\title{
Clarification in relation to the review paper "Crossbridge and filament compliance in muscle: implications for tension generation and lever arm swing"
}

\author{
Vincenzo Lombardi
}

Received: 17 January 2011/ Accepted: 15 February 2011/Published online: 9 March 2011

(C) Springer Science+Business Media B.V. 2011

In our experiments on single muscle fibres from Rana temporaria and Rana esculenta, using a combined approach of fast mechanics and $\mathrm{X}$-ray diffraction we find that the stiffness of the myosin motor is $\sim 3 \mathrm{pN} / \mathrm{nm}$ in both species and that the 1.5 times larger force per thick filament developed by Rana temporaria in isometric contraction is due to a correspondingly larger fraction of myosin heads attached to the actin filament ( $\sim 0.3$ in Rana temporaria versus $\sim 0.2$ in Rana esculenta, see for instance Decostre et al. (2005) and Piazzesi et al. (2007). In their review in J. Muscle Res. Cell Motil., Offer and Ranatunga (2010) make a contradictory interpretation of our work, and claim that the myosin motor stiffness in Rana esculenta is only $60 \%$ of that in Rana temporaria. This conclusion derives solely from their unsupported assumption that in both species the fraction of myosin heads attached to actin in isometric contraction is 0.5 . We request the publication of this clarification to avoid any misleading interpretation of our work.

\section{References}

Decostre V et al (2005) Effect of temperature on the working stroke of muscle myosin. Proc Natl Acad Sci USA 102:13927-13932

Offer G, Ranatunga KW (2010) Cross bridge and filament compliance in muscle: implications for tension generation and lever arm swing. J Muscle Res Cell Motil 31:245-265

Piazzesi G et al (2007) Skeletal muscle performance determined by modulation of number of myosin motors rather than motor force or stroke size. Cell 131:784-795
V. Lombardi $(\square)$

Laboratory of Physiology, DEB, Università di Firenze, Via G.

Sansone 1, 50019 Sesto Fiorentino, Italy

e-mail: vincenzo.lombardi@unifi.it 